\title{
In-hours Acute Home Visits by Advanced Nurse Practitioners in Primary Care: a qualitative study
}

\begin{abstract}
Background: The extension of roles within the Primary Care Team is one approach recommended to address the shortage of GPs in the UK. A key aspect of care that Advanced Nurse Practitioners (ANPs) can undertake is acute home visits. Aim: To evaluate the perspectives of Advanced Nurse Practitioners performing acute in-hours home visits in Primary Care. Methods: The research methods consist of the collection of qualitative data in eight semi-structured interviews across a Primary Care locality which were then analysed via a process of thematic analysis. Findings: Three key themes were identified: providing holistic care; engaging with the home setting; and negotiating role ambiguity. Conclusion: Practices wishing to involve ANPs in acute home visits should ensure clear definition and good understanding of the ANP role. Effective inter-professional relationships should be fostered with appropriate mentorship and clinical supervision to support ANPs in optimising their contribution to acute home visits.
\end{abstract}

Primary Care Advanced Nurse Practitioners (ANPs) are healthcare professionals who can make autonomous decisions about patient diagnosis and screening, order tests and investigations, prescribe, refer to other services such as secondary care and work with other Primary Care professionals to provide patient care (Royal College of Nursing, 2018). ANPs are now working widely as 'doctor substitutes' in Primary Care, and this substitution is considered a promising model worldwide (Van der Biezen et al, 2016). It has been highlighted that General Practice is in crisis due to a growing workload uncompensated by any increase in funding or workforce (Baird et al, 2016). General Practice is delivering care under significant pressure, not just in the surgery setting, but also in patients' private homes and residential/nursing care facilities. As the General Practice workload increases with an emphasis on carrying out more care out of hospital (NHS England, 2014) the ANP role within General Practice is expanding from chronic disease management to cover more elements of acute care. 
One key aspect of care that ANPs can undertake instead of GPs is acute home visits. Practices that have piloted new ways of working have reported some success in freeing up GP time: releasing GPs from undertaking acute home visits (as well as seeing minor ailments) was found to allow their GPs to see more complex patients (Spencer, 2016). Berg et al (2012) concluded that delegating home visits to non-GPs may improve both accessibility to visits for patients and the timeliness of the care provided.

There is very limited literature that addresses home visits, and the majority of this focuses on GP attitudes (e.g. Theile et al, 2011; Goetz et al, 2017). Collins (2018) does include ANP perspectives, but of practitioners working in out-of-hours services. No evidence was identified that explores the attitudes and experiences of the ANPs taking on home visits as part of new ways of working in in-hours Primary Care. This qualitative study therefore explores the experiences, perspectives and concerns of ANPs who are already involved in the provision of in-hours acute home visits.

\section{Aim}

The aim was to evaluate the perspectives of ANPs performing acute in-hours home visits in Primary Care.

This enabled investigation of the ANP role in relation to the distinctive nature of the home as a locus of healthcare delivery. It also provided an opportunity to evaluate perceptions of how clinician/patient interactions within home visits shapes, and is shaped by, the ANP role and professional identity. Moreover, the research offered 
insights into how ANPs negotiate with other professional groups when organising and undertaking home visits.

\section{Methods}

\section{Recruitment and sample}

The study sample was ANPs working in Primary Care who perform acute home visits. These ANPS worked for General Practices within a Clinical Commissioning Group in the West Midlands region of England. Participants were initially recruited by purposive sampling by contacting Practice Managers/GP partners of those practices within the locality which employ ANPs. The eight participants had been working as ANPs between 2 years and 16 years. [See Table 1.] (Pseudonyms have been employed within this article.) All participants were independent prescribers and practising autonomously. All were now working in NHS Primary Care, although one participant did some additional private work. All participants were White-British.

Current Royal College of Nursing standards (Royal College of Nursing, 2018) stipulate the requirement for ANPs to have a Master's qualification. However, this level of qualification was not previously stated (Royal College of Nursing, 2008) and some ANPs took on the role when a first degree was the minimum requirement. Four of the participants in this study, whose highest qualification was a first degree, were working towards a Master's qualification. All participants were working in ANP roles within their employer organisations. 
Table 1: Participant Characteristics

\begin{tabular}{|l|r|r|r|r|r|}
\hline & $\begin{array}{r}\text { Years in } \\
\text { ANP role }\end{array}$ & \multicolumn{1}{|c|}{ Age } & Gender & $\begin{array}{r}\text { Interview } \\
\text { duration } \\
\text { (minutes) }\end{array}$ & $\begin{array}{c}\text { Highest } \\
\text { Qualification }\end{array}$ \\
\hline Paula & 14 & 45 & Female & 30 & First Degree \\
\hline Clare & 3 & 42 & Female & 60 & First Degree \\
\hline Debra & 12 & 54 & Female & 70 & Diploma \\
\hline Emma & 3 & 53 & Female & 150 & Master's \\
\hline Hayley & 12 & 52 & Female & 50 & Master's \\
\hline Kate & 2 & 39 & Female & 55 & First Degree \\
\hline Joe & 4 & 43 & Male & 70 & Postgraduate \\
& & & & 60 & First Degree \\
\hline Lisa & 6 & 51 & Female & & \\
\hline
\end{tabular}

\section{Data Collection}

The data collection method employed was semi-structured interviews (King \& Horrocks, 2010). An interview schedule set out the topics to be covered and included broad questions, probing questions and follow-up questions or prompts to encourage participants to talk about specific matters of relevance in line with the research aim.

Interviews had a mean duration of approximately 50 minutes. Interviews took place either in the participants' homes or a mutually agreed location suitable for a private interview with sound recording, such as a GP surgery. The locations chosen were intended to allow the participant to relax and minimise the risk of disruption so participants could speak freely (Tod, 2010). The interviews were audio recorded and transcribed verbatim. 


\section{Data Analysis}

Thematic analysis was employed to establish key content and patterns from the data collected (Green et al, 2007). This identified commonalities (and divergence) of perspective across the interviews. Braun and Clarke (2006) outlined key stages of thematic analysis: familiarisation with the data; reading and re-reading the data in order to gain a high level of familiarity with its content; and applying codes or labels to the text to identify features of the data that are relevant to answering the research question. The principal researcher formulated an initial coding framework and a process of member-checking (Birt et al, 2016) was undertaken with a senior researcher. The framework was adjusted, where necessary, during this ongoing process. This ensured the developing analytic framework was credible and the identified themes were a coherent representation of the interview content. As an example of the thematic development, the following codes contributed to the construction of the Negotiating Role Ambiguity theme (which is articulated in the findings below): 'lack of role clarity'; 'ANP as an in-between role'; and 'medical model encroachment'.

\section{Ethical Considerations}

Permission to conduct this study was granted by Staffordshire University Research Ethics Committee. Thereafter the research was approved by the NHS Health Research Authority (HRA). All aspects of the research were performed in compliance with these ethical approvals, relevant laws and institutional guidelines. Written informed consent was obtained from all research participants. 


\section{Findings}

The data analytical process identified the following key themes: providing holistic care; engaging with the home setting; and negotiating role ambiguity. The dimensions of these themes are articulated below, drawing upon direct quotations from the interview participants. An ellipsis in square brackets highlights that some text has been removed from a quote. This is to 'clean' the text and does not alter the meaning of what was stated (Riessman, 2008).

\section{Providing Holistic Care}

ANPs identified that they could sometimes add value to visits, either through their own distinctive approach, or by doing additional tasks that other professionals might not undertake. Participants were able to give examples of times when their observation beyond expressed need resulted in a positive outcome for the patient. Below, Lisa states her view that nurses approach home visits in a different manner from doctors:

The doctor will go out and deal with the acute problem and move on whereas we go in and we see everything that needs doing. Right, this needs doing, I need to sort that out while I am in there and we're in here doing a visit; why get 3 other people in? Might as well do it while we're here. It might take another 5 or 10 minutes but it is done which in time saves time and it is better for the patient because they have had everything done all in one go - a one-stop shop. (Lisa)

It was felt that this approach is intrinsic to the ANP role, with Debra stating that it is 'natural' to offer such support. Certain duties are not supplementary or additional, but are integrated aspects of the role:

I don't think 'oh I should do this as an add-on' you just carry on because that is the way you have done it always so it comes naturally to do that, to [...] think 'right what else can I do and what else can I find out. It's just sorting them out automatically. (Debra) 
The ANPs attributed this approach to the holistic emphasis of their nurse training which, despite their additional education to become advanced practitioners, was so ingrained in them that it formed the core of their approach:

I think the nursing model is a much more holistic approach compared to the medical model. And I think that is unique to nursing [...] we just instinctively and naturally do a more holistic assessment. (Paula)

Holistic support does not only relate to engagement with patients, but also the facilitation and maintenance of positive relationships with other occupational groups. For example, interviewees also talked about cultivating relationships with care home and nursing home staff to improve communication. These relationships sometimes meant care staff felt they could contact the ANP with concerns or for advice when they might be reluctant to 'trouble' the GP:

It showed that they were willing to come and say something to me that they didn't want to go and say to the GP. (Kate)

\section{Engaging with the home setting}

All participants acknowledged the different nature of visiting a patient in their home in contrast to formal clinical settings. The home setting itself is a site that provides an opportunity for a holistic and person-centred mode of care. For example, in the following quote, Lisa highlights that the home setting could positively affect the power dynamic between the clinician and patient:

In the consultation room the GP or the consulter is the king almost and the patient comes to them. The dynamic there is completely different. In a patient's house it's their home, they've welcomed you in, they are in control more [...] so it changes the relationship between the consulter and the patient, I think. (Lisa)

The potential to engage successfully with this different setting also relates to the distinctive nature of the ANP role. For example, interviewees highlighted that their 
nursing background meant that they were attuned to the insights that a home setting might provide. The additional information a patient's home might reveal about their day-to-day life and the impact of illness was viewed as a valuable contrast with how a patient might convey themselves when they attend the surgery. This is confirmed in the following quote:

You can see a lot more that's going on and it sort of sets your nurse senses tingling. (Clare)

The nature of the home-based therapeutic relationship was also perceived to be compatible with the professional background of ANPs. Below, Hayley states her belief that doctors feel less comfortable within this context:

There is a different consultation in a patient's house and although they have never said it, I think they [the GPs] perhaps feel a little uncomfortable with that type of consultation. (Hayley)

However, it is important to recognise that respondents also highlighted challenges of engaging with home-based settings. Participants acknowledged that they felt vulnerable doing home visits not just because of their physical isolation, but because they considered themselves to be working without a "safety net" (Clare). For some this was due to a "fear you won't be equal to the task" (Paula), in part because the nature of the task was not always clear in advance. These uncertainties relating to home visits are encountered within a broader context of role ambiguity, which is addressed under the following theme.

\section{Negotiating Role Ambiguity}

A key theme raised across the interviews was the negotiation of different aspects of the ANP role, with participants perceiving that their role to some extent bridged nursing 
and medical responsibilities. For the research participants there was thus a sense of having to switch between aspects of their role, which added a tension to their experiences and a sense of not really 'belonging' to a traditional professional group anymore:

You are still a nurse but doing a role very much like a doctor's role, but you are stuck between the two. It is no man's land. It's a space between the two. (Clare)

It was also felt that some of this role confusion and weight of expectation had arisen out of a misunderstanding of what the ANPs could do:

When I first started they were almost like it was a nurse role and not a doctor's role and I think some of that was almost nervousness on the part of the GPs [...] I honestly don't believe the GPs ever knew what they were employing. (Joe)

This ambiguity and uncertainty could be a reason why the ANPs in this study seemed quite protective, even defensive, of their idea of their professional identity as nurses: "you are a nurse, you will always be a nurse to the core" (Emma). Despite all the participants having worked hard to achieve their ANP status this did not mean they had eschewed 'nurse status'. "I am a nurse and I will always be a nurse" (Hayley).

It was also identified, however, that there could be benefits to this new status. Lisa intimated that the negotiation of roles could lead to advantages or disadvantages for a particular professional group, but she ultimately asserted that decision-making should be for the benefit of the whole practice:

I do see [the ANP role] as a link between doctors and nurses, sort of midway and $[. .$.$] we use it for the benefit of the practice. (Lisa)$ 


\section{Discussion}

The findings in this research highlight the potential of the ANP role to undertake home visits successfully. There is a clear sense that the orientation of the nursing role means that ANPs are well equipped to engage with visits, and to deliver a holistic mode of support in this setting. This, in part, seems to reflect the ethos of nursing and the training that has been undertaken (Nursing and Midwifery Council, 2020). This reinforces the sense that ANP is a distinctive role: grounded in depth and breadth of knowledge and skills; the degree of information synthesis required; and the complexity of the interventions addressed (Hamric, 2000).

The exploration of perspectives on home visits generated insights into broader aspects of ANP role and professional identity. Most of the interviewees spoke about the role transition from nurse to ANP, being prepared to revert to the nurse role when needed. Billay et al (2015) explored the experience of transitioning from the role of nurse to that of ANP and recognised that the process involved three stages of student ANP, newly qualified ANP and established ANP. The different stages are anxiety-provoking, then messy and uncertain before the ANP feels they are truly established in their role. Until this happens, they are in a liminal space. This adjustment of role is also likely to impact upon other working relationships. Harris et al. (2016) noted that interprofessional collaboration, such as that between ANPs and GPs, does challenge professionals' ideas of their roles as well as their work practices.

Mentorship and clinical supervision have a key role to play in supporting ANPs to negotiate this transition process. It is important to recognise how practical aspects of the role, and the development of professional identity development are intrinsically 
linked and can be supported by experiential learning. "A shared professional identity within ANPs' communities of practice enables collective awareness of values and practices inherent in the advanced practitioner role" (Kerr \& Macaskill, 2020: 564).

It was evident from the ANPs in this study that their nursing background was a source of pride and highly valued as enriching their skills and therefore what they could offer to patients on home visits. It has been noted that interprofessional collaborations do challenge professionals' ideas of their roles, as well as their work practices and interactions with others (Harris et al, 2016; Anderson, 2017). In addition, Main et al (2007) noted discomfort from ANPs when they tried to fit their nursing into a medical model. This might explain why the ANPs in this study made such clear appeals to the formation of nursing identities and roles.

Within this research, the expansive nature of the role (including home visits) seemed to create additional pressures, with ANPs having to 'be everything to everyone'. This is in line with other studies that have explored the transition from nurse to ANP. For example, it has been noted that role extension can lead to role confusion and role overlap which can result in stress (McKenna et al 2014). The sense of being situated between the two roles of nurse and doctor appears to play a significant part in this confusion. As noted in the final quote in the findings from Lisa, this situation could generate positive outcomes: while ambiguity could cause practitioners to retreat to the reassurance of a traditional professional identity (Anderson, 2017), a flux in role parameters can be positively harnessed. This could then be converted into a positive mode of flexibility, under which collaboration between professional groups is enhanced. This flexibility must, however, be reconciled with the maintenance of a core 
nursing identity: even though there is some overlap in orientation between ANP and medic, the ANP role must remain implemented within the context of nursing (McDonald, 2006). The extent to which ANPs feel their role is an extension of the nursing role, or constitutes a space between medicine and nursing, should be a fruitful area for further research.

\section{Study Limitations}

A small qualitative study with a relatively homogenous sample is not intended to be generalisable. For example, the sample was White-British only, and predominantly female. However, although the study participants were all working in Primary Care, some of their experiences are transferable to ANPs working in other settings who are undergoing, or have already undertaken, the transition from nurse to ANP. The findings are likely to be less relevant to Advanced Clinical Practitioners who do not have a nursing background. The research did not seek GP perspectives on the ANP role. This could be usefully incorporated into a future study.

\section{Conclusion}

This article has demonstrated ways in which ANPs can enrich home visits and offer holistic care to patients, while sharing the GP workload. The key findings of this study are that ANPs can enhance acute home visits by using their nursing skills and professional networking abilities. ANPs can add value by undertaking additional tasks and activities during acute home visits.

Confusion about the ANP role and lines of accountability continues to impact upon the process of utilising ANPs for acute home visits. A key challenge is thus to convert 
ambiguity into a positive flexibility. The liminality between roles potentially creates a space within which flexible, but resilient, professional identities can be forged. This, however, is dependent on the ANP role itself being well defined, otherwise a sense of ambiguity and confusion could reduce levels of personal confidence, and accordingly the quality of professional relationships. ANPs continuing to contribute with acute home visits provides a means towards which this enhanced collaborative framework can become embedded in ongoing practice.

Practices wishing to involve ANPs in acute home visits should thus ensure good understanding and clear definition of the ANP role. Good inter-professional relationships should be fostered with appropriate mentorship and clinical supervision to support the expansion of ANPs' clinical skills to optimise their contribution to acute home visits. This can help to ensure that patient care and the needs of the service are prioritised ahead of the needs or preferences of any particular professional group. 
Keywords: Advanced Nurse Practitioner; holistic care; home visits; professional identity

\section{Key points:}

The aim of this qualitative research study was to evaluate the perspectives of ANPs performing acute in-hours home visits in Primary Care.

ANPs are well equipped to engage with acute home visits, and to deliver a holistic mode of support in this setting.

A challenge of the ANP role is a sense of no longer really 'belonging' to a traditional professional group.

Appropriate mentorship and clinical supervision can help ANPs to optimise their contribution within acute home visits.

\section{CPD Reflective Questions:}

What are the key differences between the delivery of patient care in clinical and home settings?

Reflect upon your ability to communicate with different professional groups. What are the potential barriers to communication and how can they be overcome?

Consider what training or career development would be required for you (or members of your team) to become an ANP. 


\section{References}

Anderson, H. (2017) 'Professional identity and the advanced nurse practitioner in Primary Care: A qualitative study'. PhD thesis, University of York.

Baird, B., Charles, A., Honeyman, M., Maguire, D. \& Das, P. (2016) Understanding pressures in general practice. London: King's Fund. Available at: www.kingsfund.org.uk/sites/default/files/field/field publication file/Understandi ng-GP-pressures-Kings-Fund-May-2016.pdf (last accessed 16th December 2020).

Berg van den, N., Heymann, R., Meinke, C., Baumeister, S. E., Flessa, S., \& Hoffmann, W. (2012). Effect of the delegation of GP-home visits on the development of the number of patients in an ambulatory healthcare centre in Germany. BMC Health Services Research,12: 355.

Biezen dan der, M., Adang, E., Van Der Burgt, R., Wensing, M. \& Laurant, M. (2016) The impact of substituting general practitioners with nurse practitioners on resource use, production and health-care costs during out-of-hours: a quasiexperimental study, BMC Family Practice, 17: 132.

Billay, D., Myrick, F., \& Yonge, O. (2015). Preceptorship and the nurse practitioner student: navigating the liminal space. Journal of Nursing Education, 54(8): 430-437.

Birt, L. Scott, S., Cavers, D., Campbell, C. \& Walter, F. (2016) Member checking: a tool to enhance trustworthiness or merely a nod to validation? Qualitative Health Research, 26(13): 1802-1811.

Braun, V. \& Clarke, V. (2006) Using thematic analysis in psychology. Qualitative Research in Psychology, 3(2): 77-101. 
Collins, D. (2018) Assessing the effectiveness of advanced nurse practitioners undertaking home visits in an out of hours urgent Primary Care service in England. Journal of Nursing Management, 27(2): 450-458.

Goetz, K., Kornitzky, A., Mahnkopf, J. \& Steinhäuser, J. (2017) At the dawn of delegation? Experiences and attitudes of general practitioners in Germany - a questionnaire survey. BMC Family Practice, 18: 102.

Green, J. et al. (2007) Generating best evidence from qualitative research: The role of data analysis. Australian and New Zealand Journal of Public Health, 31(6), 545-550.

Hamric, A. (2000). A definition of advanced nursing practice. In A. Hamric, J. Spross, \& C. Hanson (Eds.), Advanced Nursing Practice: An integrative approach (pp. 53-73). Philadelphia, PA: W. B. Saunders.

Harris, M.F. et al. (2016) Interprofessional teamwork innovations for primary health care practices and practitioners: evidence from a comparison of reform in three countries. Journal of Multidisciplinary Healthcare, 29(9): 35-46.

Kerr, L. \& Macaskill, A. (2020) The journey from nurse to advanced nurse practitioner: applying concepts of role transitioning. British Journal of Nursing, 29(10).

King, N. \& Horrocks, C. (2010) Interviews in Qualitative Research. London: Sage.

Main, R., Dunn, N., \& Kendall, K. (2007) Crossing professional boundaries: Barriers to the integration of nurse practitioners in primary care. Education for Primary Care, 18(4): 480-487.

McDonald, J. (2006) Advanced practice nursing: unification through a common identity. Journal of Professional Nursing, 22(3):172-179. 
McKenna, H., Pajnkihar, M. \& Murphy, F. (2014) Fundamentals of Nursing Models, Theories and Practice. Oxford: Wiley Blackwell.

NHS England (2014) 'Five Year Forward View', available at:

https://www.england.nhs.uk/wp-content/uploads/2014/10/5yfv-web.pdf (last accessed 16 December 2020).

Nursing and Midwifery Council (2020) ‘NMC Strategy 2020-2025’. London: Nursing and Midwifery Council, available at:

https://www.nmc.org.uk/globalassets/sitedocuments/strategy/nmc-strategy2020-2025.pdf (last accessed 16 December 2020).

Riessman, C. K. (2008) Narrative Methods for the Human Sciences. London: Sage

Royal College of Nursing (2008) 'Advance Nurse Practitioners - an RCN guide to the advanced nurse practitioner role, competencies and programme accreditation', available at: http://aape.org.uk/wpcontent/uploads/2015/02/RCN-ANP-quidance-document-2008.pdf (last accessed 19th January 2021).

Royal College of Nursing (2018) 'Standards for Advanced Level Nursing Practice', available at: https://www.rcn.org.uk/library/subject-guides/advanced-nursingpractice (last accessed 16 ${ }^{\text {th }}$ December 2020).

Spencer, M. (2016) 'Skill mix: how paramedics can help general practice', GP Online, 26 ${ }^{\text {th }}$ May 2016, available at: https://www.gponline.com/skill-mixparamedics-help-general-practice/article/1396392 (last accessed 16th December 2020).

Theile, G. Kruschinski, C., Buck, M., Müller, C. \& Hummers-Pradier, E. (2011) Home visits - central to primary care, tradition or an obligation? A qualitative study. BMC Family Practice, 12: 24. 
Tod, A. (2010) 'Interviewing' in: Gerrish, K. \& Lacey, A. (eds.) The Research Process in Nursing. [6th ed.] pp. 345-357.Chichester: Wiley-Blackwell. 